\title{
DISTANCE SPHERES IN COMPLEX PROJECTIVE SPACES
}

\author{
ALAN WEINSTEIN ${ }^{1}$
}

\begin{abstract}
Distance spheres in complex projective spaces are counterexamples to the odd-dimensional extension of a lemma of Klingenberg.
\end{abstract}

Klingenberg proved in 1959 [4] that if $M$ is a compact, simply connected, even-dimensional manifold whose sectional curvatures lie in the interval $(0, K]$, then the length of any closed geodesic in $M$ is at least $2 \pi K^{-1 / 2}$. He then ([5], [6]) extended the result to odd-dimensional manifolds in the case where the curvature lies in [(1/4)K, K]. In 1962, Berger [1] gave examples of metrics on $S^{3}$, with curvatures lying in $[\delta K, K]$ for all $\delta \in$ $(0,1 / 9)$ and with closed geodesics of length less than $2 \pi K^{-1 / 2}$. Later Chavel [2] constructed similar metrics on all odd-dimensional spheres. The purpose of the present note is to point out that the examples of Berger and Chavel occur "in nature" as distance spheres in complex projective space.

Consider $C P^{n}$ with the standard metric having sectional curvatures in the interval $[1 / 4,1]$ and diameter $\pi$. Fix $p \in C P^{n}$ and let $S_{r}$ be the set of points having distance $r$ from $p$. For $r \in(0, \pi), S_{r}$ is a $2 n-1$ dimensional sphere on which the isotropy group $U(n)$ of $p$ acts transitively and isometrically. The central subgroup of $U(n)$ generates a Killing vector field of constant length on $S_{r}$ whose orbits are closed geodesics of length $2 \pi \sin r$. Using Gromoll's formula [3, p. 361] for the curvature of distance spheres, one finds that the maximum and minimum sectional curvatures of $S_{r}$ are $(1+4 u) / 4 u$ and $1 / 4 u$ respectively, where $u=\tan ^{2}(r / 2)$. Now $2 \pi \sin r$ becomes less than $2 \pi((1+4 u) / 4 u)^{-1 / 2}$ when $u>2$, i.e. when $r$ is greater than $2 \arctan \sqrt{ } 2=\arccos (-1 / 3)$. The ratio $1 /(1+4 u)$ of minimum to maximum curvature drops below $1 / 9$ when $u$ rises above 2 .

That the distance spheres in $C P^{n}$ are isometric to the examples of Berger and Chavel follows from the fact that both spaces are homogeneous spaces

Received by the editors October 19, 1972 and, in revised form, January 30, 1973. AMS (MOS) subject classifications (1970). Primary 53C20, 53C30.

Key words and phrases. Complex projective space, geodesics, sectional curvature.

${ }^{1}$ Supported in part by a fellowship from the Alfred P. Sloan Foundation. 
of $U(n)$, with metric foreshortened in the direction of the vector field generated by the central subgroup.

Distance spheres in quaternionic projective spaces and the Cayley plane are further counterexamples to the odd-dimensional extension of Klingenberg's lemma. The critical curvature ratio in these cases is still $1 / 9$.

\section{REFERENCES}

1. M. Berger, On the diameter of some Riemannian manifolds, Technical Report, University of California, Berkeley, 1962.

2. I. Chavel, A class of Riemannian homogeneous spaces, J. Differential Geometry 4 (1970), 13-20. MR 42 \#5185.

3. D. Gromoll, Differenzierbare Strukturen und Metriken positiver Krümmung auf Sphären, Math. Ann. 164 (1966), 353-371. MR 33 \#4940.

4. W. Klingenberg, Contributions to Riemannian geometry in the large, Ann. of Math. (2) 69 (1959), 654-666. MR 21 \#4445.

5. —_ Über Riemannsche Mannigfaltigkeiten mit positiver Krümmung, Comment. Math. Helv. 35 (1961), 47-54. MR 25 \#2559.

6. —_, Über Riemannsche Mannigfaltigkeiten mit nach oben beschrankter Krümmung, Ann. Mat. Pura Appl. (4) 60 (1962), 49-59. MR 28 \#556.

School of Mathematics, Institute for Advanced Study, Princeton, New JERSEY 08540

Current address: Department of Mathematics, University of California, Berkeley, California 94720 